\title{
Diseño Y Simulación De Un Robot Humanoide De Servicio Para Los Laboratorios De Control - Automatización Y Telecomunicaciones De La Universidad Nacional Tecnológica De Lima Sur
}

\author{
Ricardo J. Palomares Orihuela dol , Julián Antonio Ruiz Sandoval, Alexander Javier Romero \\ Cuellar y Fredy Campos Aguado \\ Universidad Nacional Tecnológica de Lima Sur, Lima, Perú
}

Recibido: 15/11/2020 Revisado: 21/11/2020 Aceptado: 26/11/2020 Publicado: 28/01/2021

\section{RESUMEN}

La presente investigación comprende el diseño y simulación de un robot humanoide de servicio, mediante la selección del material y dimensiones de su estructura, componentes electrónicos y servidor, así como, la selección de los sensores y actuadores que le permitirán interactuar con el medio que lo rodea, percibiendo del entorno y realizar una acción. Así mismo, se realizó el diseño y simulación de su sistema informático e interfaz de reconocimiento facial. Se validó mediante la simulación dinámica en 3D del robot humanoide usando un software de ingeniería. Los resultados favorables se obtuvieron a partir de la conmutación de la PC con el sistema operativo Windows y un dispositivo Raspberry pi 3B+ que se desempeña bajo un sistema operativo GNU/LINUX en la distribución Raspbian El entrenamiento del algoritmo de reconocimiento facial y la trasferencia de datos se probó exitosamente, obteniendo resultados favorables. Finalmente, se contribuyó con la comunidad científica y estudiantil al brindar una plataforma para futuras investigaciones.

Palabras claves: robot, humanoide, simulación, inteligencia artificial, Raspberry Pi.

\begin{abstract}
The present investigation includes the design and simulation of a humanoid service robot, by selecting the material and dimensions of its structure, electronic components and server, as well as the selection of sensors and actuators that will allow it to interact with the environment that uses it. surrounds, perceiving the environment and performing an action. Likewise, the design and simulation of its computer system and facial recognition interface was carried out. It was validated by dynamic 3D simulation of the humanoid robot using engineering software. The favorable results were obtained from the switching of the PC with the Windows operating system and a Raspberry pi 3B + device that works under a GNU / LINUX operating system in the Raspbian distribution. The training of the facial recognition algorithm and the transfer of data was successfully tested, obtaining favorable results. Finally, it contributed to the scientific and student community by providing a platform for future research.
\end{abstract}

BIOTECH \& ENGINEERING Untels. Ene - jun.1(1), 2021; ISSN: 2788 - 4295; $63-81$ DOI: https://doi.org/10.52248/eb.Vol1lss01.1 
Keywords: robot, humanoid, simulation, artificial intelligence, Raspberry Pi.

\section{INTRODUCCIÓN}

\section{Planteamiento del Problema}

Los robots humanoides son aplicados en universidades extranjeras con fines educativos e interacción maximizando el rendimiento de diversos procesos realizando una tarea específica de manera automatizada y/o autónoma. Los humanoides son plataformas muy efectivas para probar el desempeño de algoritmos y estrategias de control, así como de sistemas de programación y de percepción avanzada.

Universidades americanas y europeas incluyen robots humanoides como parte de sus procesos educativos, enseñanza - aprendizaje, así como en aquellos que puedan interactuar con los estudiantes, como son en las bibliotecas, comedores, así como con los docentes, a través del apoyo en el dictado de la cátedra, así como en los ambientes de laboratorio.

Debido al desarrollo de robots humanoides de servicio para el apoyo en tareas cotidianas y académicas, se plantea la necesidad de desarrollar la presente investigación a través de un robot humanoide Asistente con la capacidad de realizar la función de apoyo en la administración de materiales, instrumentos, manejo base de datos, conectividad a la nube, así como plataforma de investigación para futuros proyectos.

\section{Formulación del problema}

\section{Problema general}

¿Cómo brindar una alternativa automatizada que permita mejorar el servicio académico y administrativo en los Laboratorio de la Escuela Profesional de Ingeniería Electrónica y Telecomunicaciones de la Universidad Nacional Tecnológica de Lima Sur?

\section{Problemas específicos}

¿Qué tecnología permitirán contar con un sistema mecánico - electrónico que mejore el servicio académico y administrativo?

¿Qué tecnología permitirán contar con un sistema de red y Cloud local que mejore el servicio académico y administrativo?

¿De qué manera será posible verificar el funcionamiento de un sistema automatizado que mejore el servicio académico y administrativo?

\section{Objetivos del proyecto}

BIOTECH \& ENGINEERING Untels. Ene - jun.1(1), 2021; ISSN: 2788 - 4295; $63-81$ 


\section{Objetivo general}

Diseñar y simular un Robot Humanoide de Servicio para los Laboratorios de Control Automatización y Telecomunicaciones de la Universidad Nacional Tecnológica de Lima Sur.

\section{Objetivos específicos}

Diseñar el sistema mecánico - electrónico para un robot humanoide.

Diseñar el sistema de red y Cloud local del robot humanoide

Simular el sistema mecánico, electrónico, red y Cloud local para un robot humanoide

\section{MARCO TEÓRICO}

\section{Robot Humanoides de Servicio}

La Federación Internacional de Robótica (IFR) define a un robot de servicio como: "aquel que trabaja de manera parcial o totalmente autónoma, desarrollando servicios utilices para el bienestar de los humanos y equipos. Excluyendo operaciones de manufactura. Pueden ser móviles y con capacidad de manipulación".

Cuando los robots de servicios tienen contacto directo con las personas e interactúan con ellas se les denomina robots asistentes, por lo que en su diseño es importante tener en cuenta: su autonomía, la seguridad y la facilidad de integración en el entorno. De esta manera, en un futuro, cualquier persona personalizaría a su propio robot para que lo asista. La mayoría de los investigadores en este campo apuntan a los robots que intentan simular la forma humana, es decir, los robots humanoides. (Cabrera, 2013)

\section{Interacción Hombre-Robot (HR)}

La interacción hombre-robot (IHR) juega un papel importante en cualquier sistema de robótica teniendo en cuenta que todavía no existe un robot con capacidad totalmente autónoma. Incluso si esta meta de la autonomía completa se alcanzara, el papel humano y el nivel de interacción variarán, pero el hombre seguirá siendo una parte del sistema (Khamis, 2003).

BIOTECH \& ENGINEERING Untels. Ene - jun.1(1), 2021; ISSN: 2788-4295; 63 - 81 
La IHR puede definirse como el estudio de los seres humanos, los robots, y la forma en que uno influye en el otro (Fong, 2002). Sheridan ha mencionado que uno de los desafíos de la interacción hombre-robot es proveer a los seres humanos y a los robots modelos de uno al otro (Sheridan, 1997).

\section{Relaciones Hombre-Robot}

La interacción humana y la autonomía del robot son las funciones claves que pueden ampliar el uso del robot en la vida diaria. Hoy en día, la mayoría de los robots disponibles pueden interactuar únicamente con sus creadores o con un grupo pequeño de individuos especialmente entrenados. El objetivo final de las investigaciones en robótica es desarrollar un robot que pueda interactuar con seres humanos y participar en la sociedad humana. Este tipo de robot debe tener interfaces efectivas, intuitivas y transparentes con nivel alto de autonomía por lo cual el robot será capaz de sobrevivir en situaciones diferentes. (Khamis, 2003).

\section{Estado del Arte}

Se revisaron publicaciones relacionadas al tema de la investigación, los cuales se muestran a continuación:

Blanco. D, S. Ansari, C. Castejon, B. López, L. Moreno (2005), "Manfred: Robot Antropomórfico de servicio fiable y seguro para operar en entornos humanos", Revista Iberoamericana de Ingeniería Mecánica, Vol. 9, º3, pp. 33-48, España.

Hernández. C, Montoya. C (2007), "Diseño de un sistema emulando el movimiento articulado de una mano, brazo y antebrazo", dirigido por Oliver. M, Tesis de Maestría en Ingeniería Mecatrónica, CENIDET, México.

Loza. E (2008), "Brazo Robótico controlado por una computadora en un ambiente de realidad virtual”, Tesis Maestría en Ciencias en Tecnología de Computo, Instituto Politécnico Nacional, México.

Jardón. A (2006), "Metodología de diseño de robots asistenciales. Aplicación al robot portátil ASIBOT”, Tesis Doctoral dirigido por A. Giménez, C. Balaguer. Universidad Carlos III de Madrid, España.

En el 2013, egresados de la Escuela Profesional de Ingeniería Mecatrónica de la Universidad Ricardo Palma obtuvieron su título con la Tesis "Diseño e implementación de un robot humanoide asistencial controlado por computador para aplicaciones en pacientes parapléjicos"

Un grupo de investigadores de la Pontificia Universidad Católica del Perú - PUCP se encuentra perfeccionando un robot humanoide, llamado IOmi, que será la anfitriona en el Complejo de Innovación Académica, con la que se presentaron en el Concurso Nacional de Invenciones 2015.

BIOTECH \& ENGINEERING Untels. Ene - jun.1(1), 2021; ISSN: 2788 - 4295; $63-81$ 


\section{METODOLOGÍA}

\section{Consideraciones previas a su diseño}

A continuación, se muestra las características fundamentales que debe reunir el robot humanoide:

El robot humanoide debe asegurar la seguridad del usuario dado que existe una estrecha interacción, incluso contacto, con el usuario.

Debe permitir a través de su arquitectura la interacción natural con los estudiantes.

Deben emplear tecnologías o dispositivos de fácil acceso, es decir, utilizables por cualquier usuario, sin necesidad de formación, o de superar una elevada curva de aprendizaje.

\section{Arquitectura de un robot humanoide.}

La figura 1 muestra los sistemas que se consideran en el diseño de un robot humanoide, un sistema mecánico que incluye un subsistema locomotor, así como del sistema electrónico que incluye un subsistema de conexión a un servidor web.

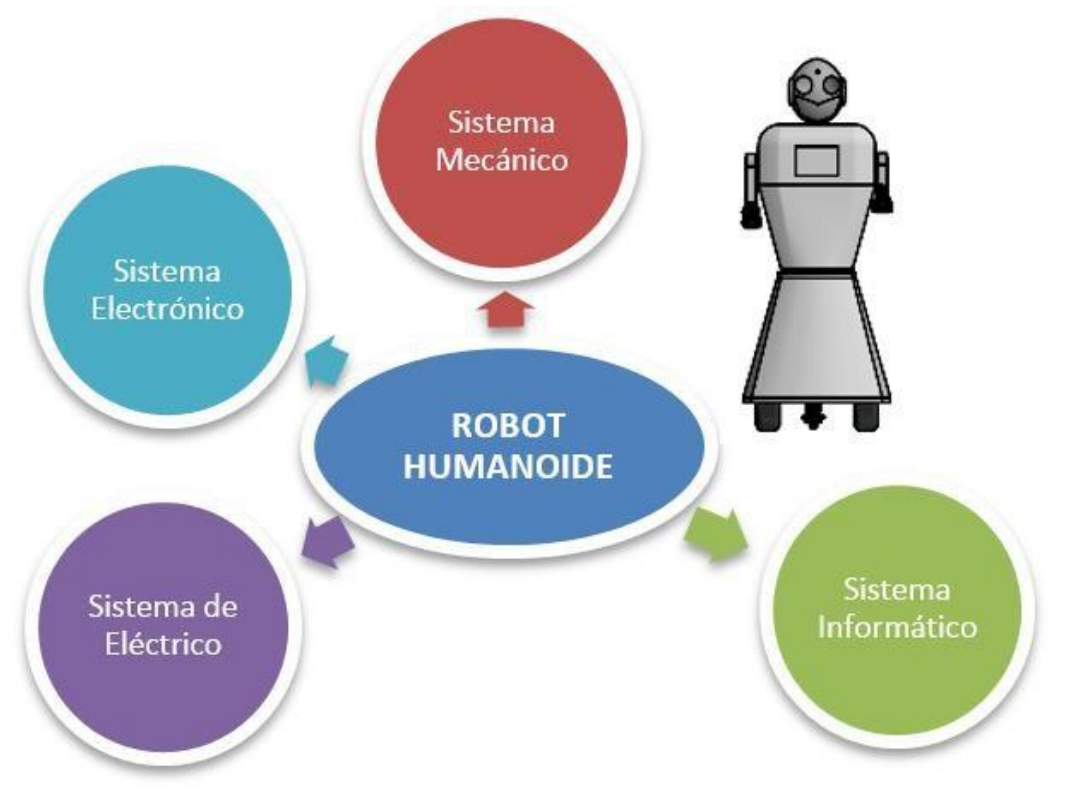

Figura 1. Arquitectura de los sistemas de un Robot Humanoide. Fuente: Elaboración propia

Diseño y simulación del Sistema Mecánico del robot.

BIOTECH \& ENGINEERING Untels. Ene - jun.1(1), 2021; ISSN: 2788-4295; $63-81$ 
El desempeño del robot humanoide requiere de un diseño mecánico particular de acuerdo a la aplicación. La estructura del sistema mecánico del robot tiene las siguientes partes:

Base: aloja el sistema locomotor.

Tronco: donde están instaladas las tarjetas electrónicas y la pantalla.

Los brazos: que le permite al robot manipular objetos pequeños de laboratorio.

La cabeza: están alojados la cámara de video y el sistema de reproducción de audio.

Para la estructura de soporte del robot humanoide se seleccionó al aluminio por su dureza, y baja densidad de $2.669 \mathrm{~g} / \mathrm{cm} 3$. En la estructura externa se usó plástico PLA y ABS, por su baja conductividad térmica y eléctrica, además por su facilidad de uso con impresoras 3D. La estructura del sistema mecánico ha sido diseñada usando el Software SolidWorks. En la figura 2, se presenta el diseño mecánico del robot humanoide, destacando su altura de $1650 \mathrm{~mm}$ y un ancho total en la base de $606 \mathrm{~mm}$. 


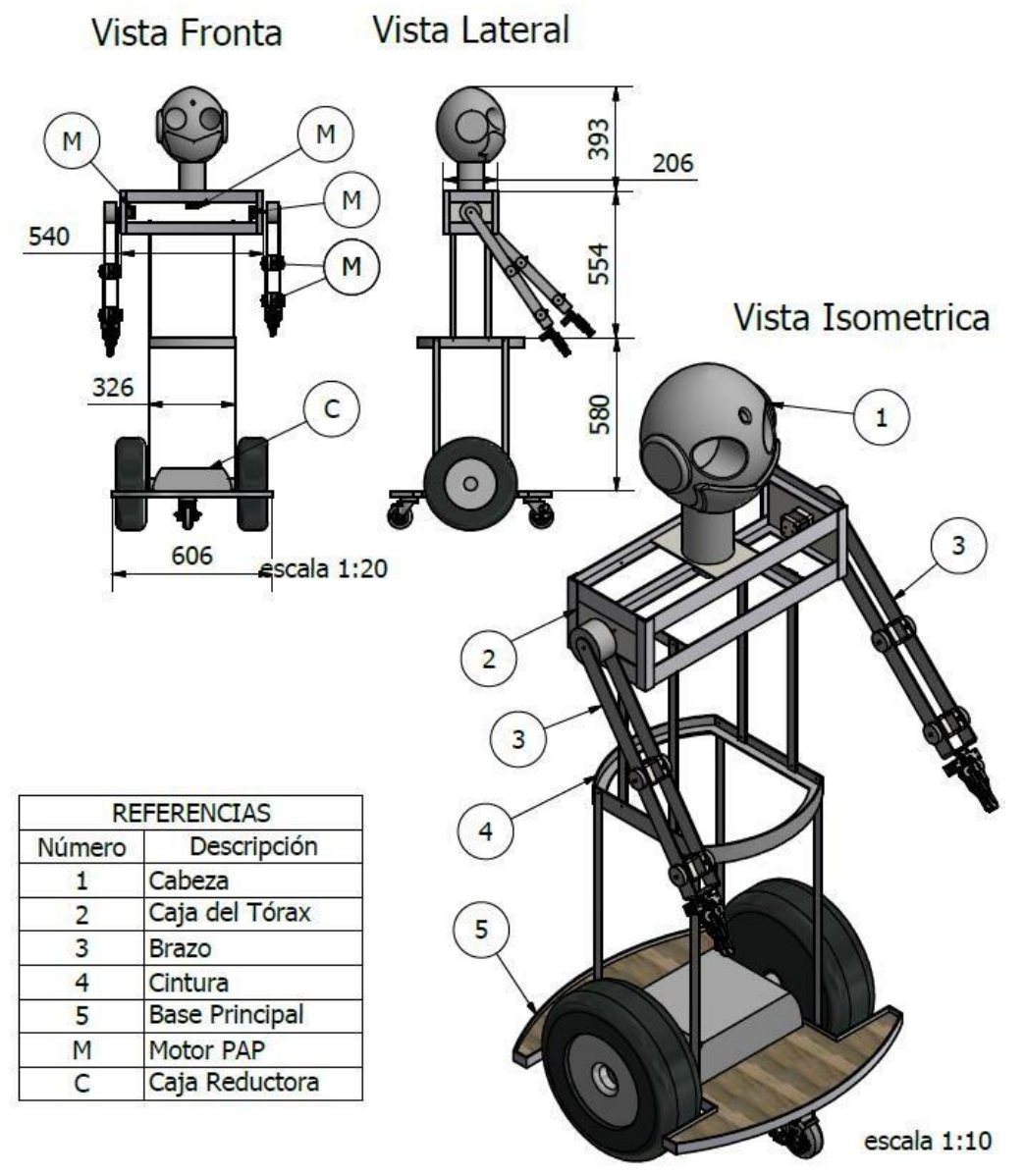

Figura 2. Diseño mecánico del robot humanoide. Fuente: Elaboración propia

\section{Diseño y simulación del Sistema electrónico}

En la figura 3 se muestra de manera gráfica el sistema electrónico, con el procesador, un controlador, cámara detectora de imágenes, sensores de proximidad de obstáculos y personas, actuadores, así como un Servidor de datos. 


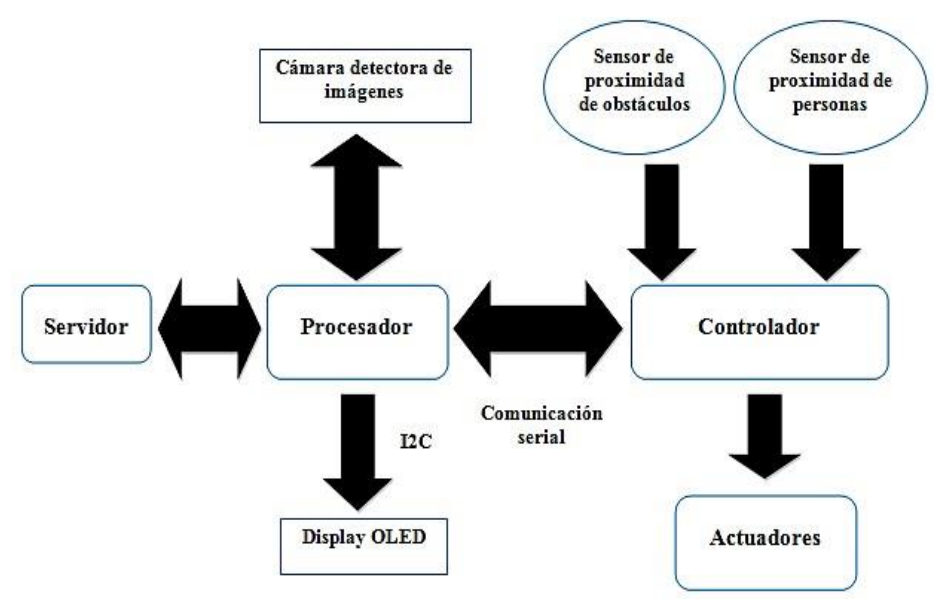

Figura 3. Esquema general del diseño del sistema electrónico

Fuente: Elaboración propia

Se seleccionó el procesador Raspberry, que procesa la información de la cámara y recibe los archivos entrenados de reconocimiento facial enviados por el Servidor vía WI-FI; el controlador arduino Mega 2560, recibe la señal procesada del Raspberry y acciona los motores del robot, así mismo recibe información de los sensores de proximidad y PIR y evite colisión con objetos; pantalla LCD para mostrar la información solicitada, todo lo cual se muestra en la figura 4.

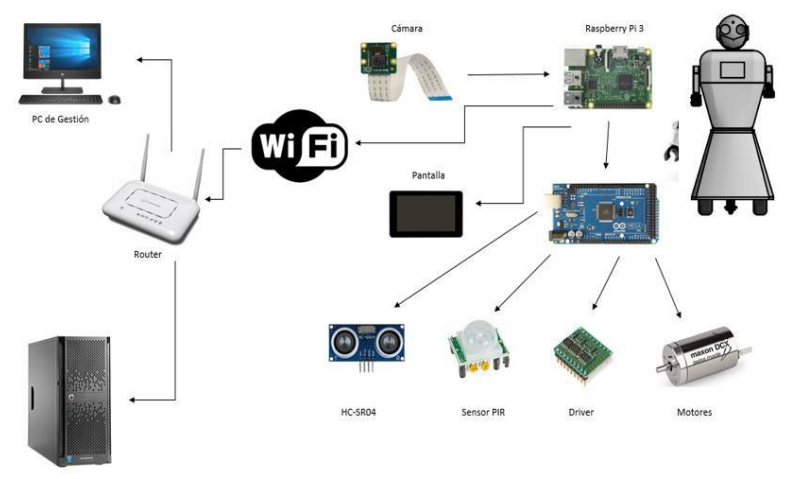

Figura 4. Esquema general del robot humanoide y su entorno Fuente: Elaboración propia 


\section{Diseño del Sistema de Red y Cloud local}

Se requiere de una red independiente que se bifurque de la red del Laboratorio de Control y Automatización, mediante un Servidor, una computadora de gestión y el Robot Humanoide.

El robot humanoide se comunica vía Wi-Fi al Servidor de manera bidireccional intercambiando información entre sí, todo ello conmutando por el protocolo SFTP (FTP over SSH) el cual se encarga de encriptar la información y proteger la identidad de los estudiantes.

El servidor almacena la información de una red neuronal y se encarga de realizar el entrenamiento de las mismas y transfiere el resultado al Robot Humanoide, y realiza el reconocimiento del rostro del estudiante y por ende de todos los usuarios del Laboratorio. En la figura 5 se muestra un esquema del Sistema de Red y Cloud Local.

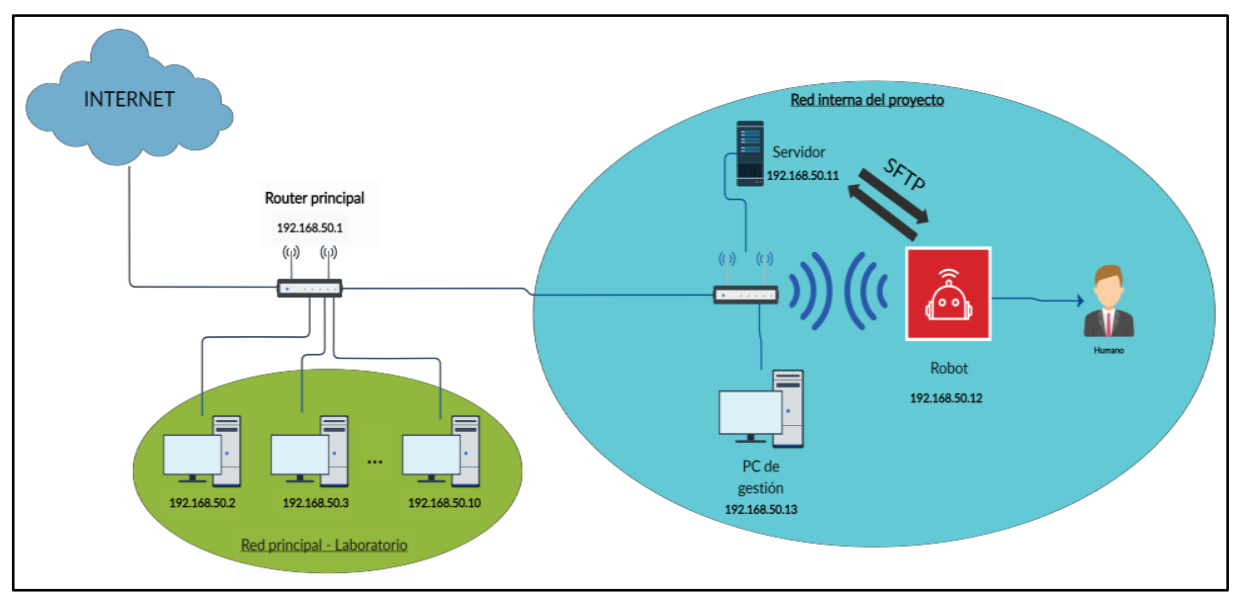

Figura 5. Esquema general del Sistema de Red y Cloud Local

Fuente: Elaboración propia

En la Figura 6 se muestra el Servidor adquirido, HP ProLiant ML150 Gen9, Intel Xeon E5-

2609v4 1.70GHz, con 8GB de RAm y Disco Duro LFF SATA de 1 Tera de capacidad.

BIOTECH \& ENGINEERING Untels. Ene - jun.1(1), 2021; ISSN: 2788-4295; 63 - 81 


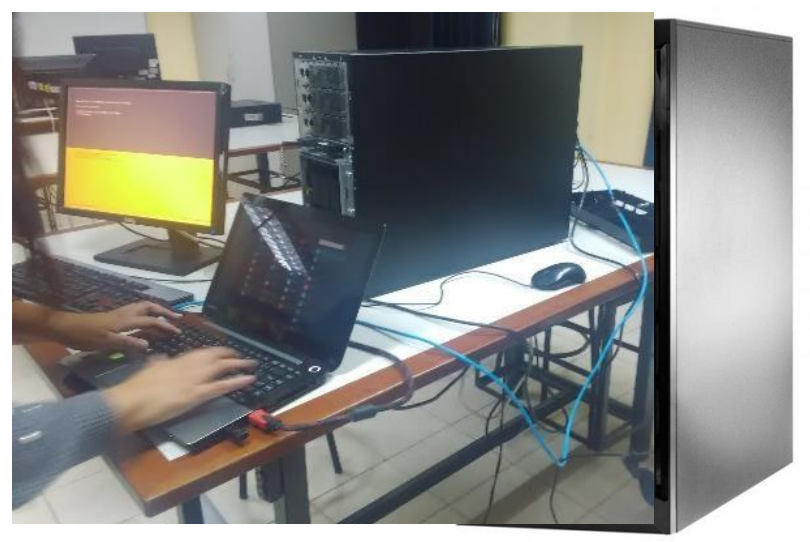

Figura 6. Servidor HP adquirido para la investigación

Fuente: Elaboración propia

El Servidor HP está configurado con el sistema operativo Ubuntu de GNU/LINUX, la plataforma de trabajo se puede visualizar a través del entorno de virtualización VMWARE. La instalación de softwares se realizó utilizando las computadoras y red del laboratorio, y se define una ubicación segura del servidor al interior del Laboratorio, tal como se muestra en la Figura 7.
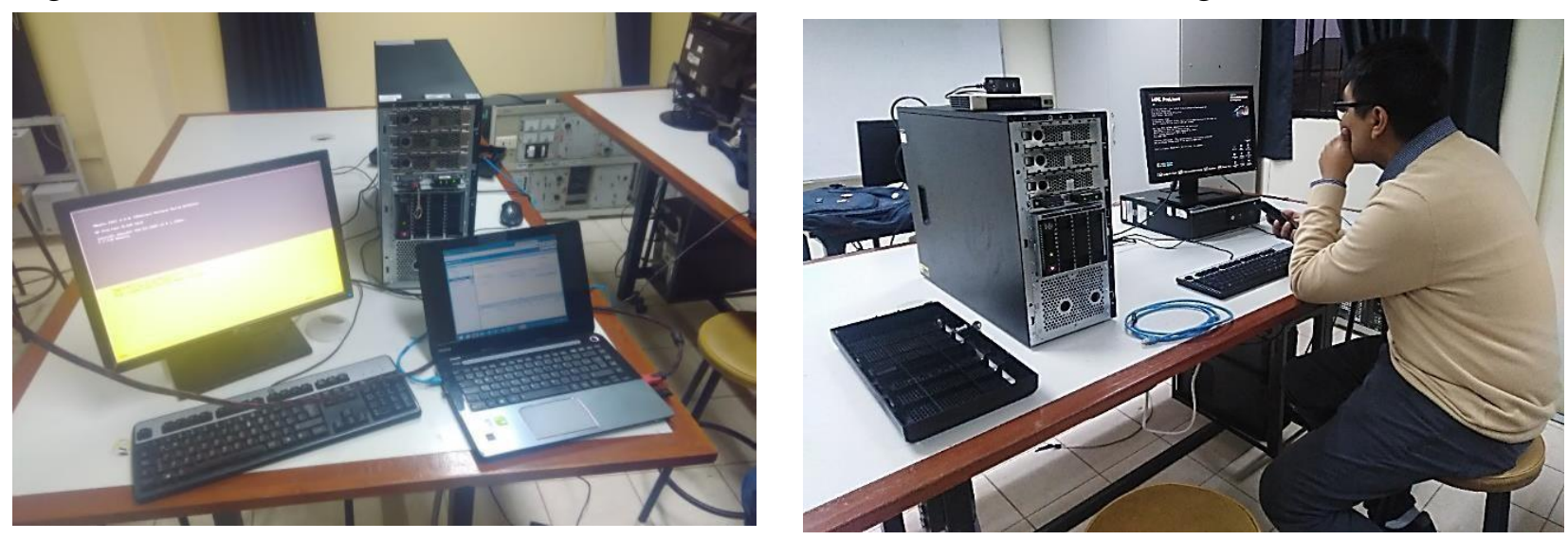

Figura 7. Servidor en actividad

Fuente: Elaboración propia (2019)

\section{Sistema de detección facial}

En el proceso de elaboración de la primera etapa del algoritmo de detección facial se utiliza el lenguaje Python 3 programado mediante el editor de texto Notepad ++ como se puede apreciar en la figura 8 instalado en el sistema operativo Windows utilizando la versión de Python 3.7.4. 


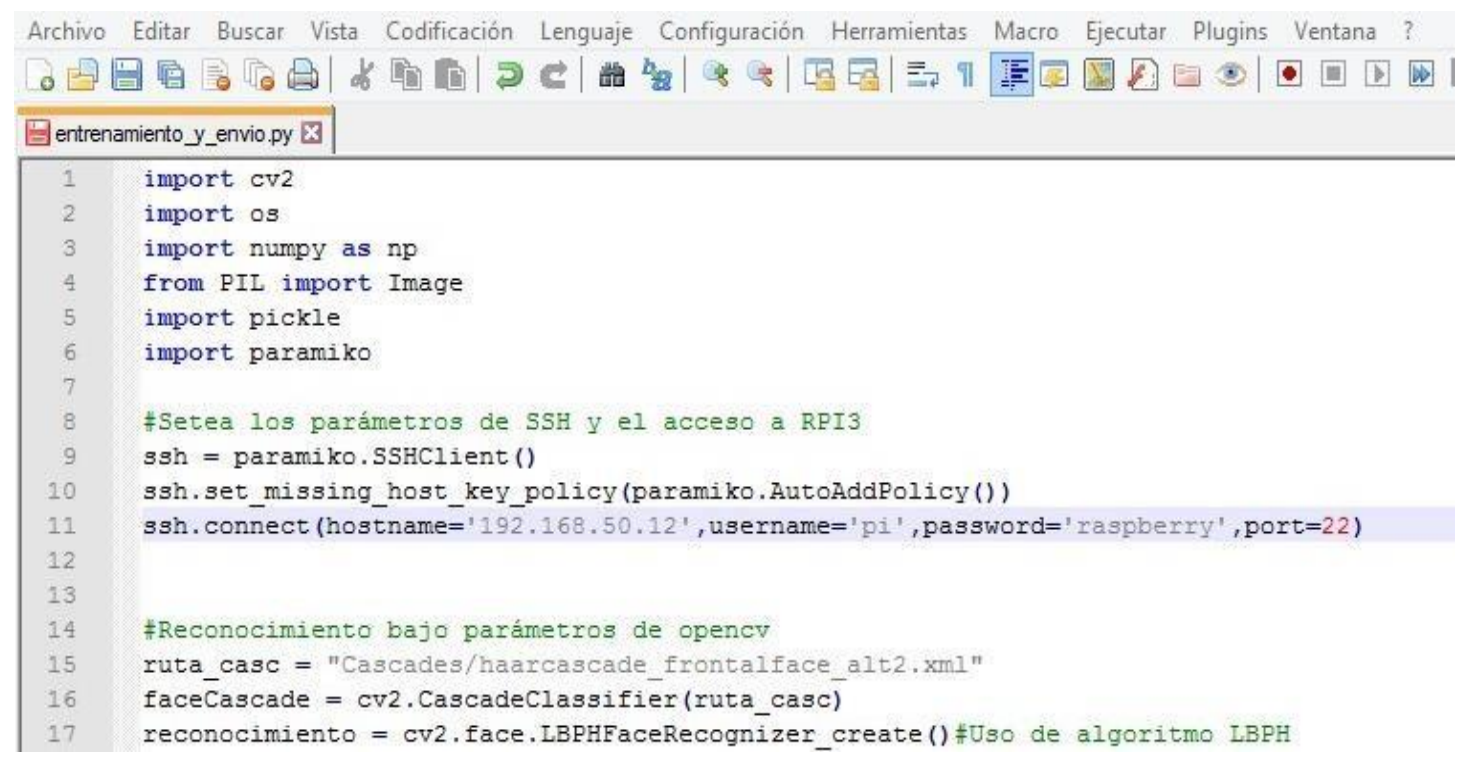

Figura 8. Interfaz de Notepad ++ en Windows. Fuente: Elaboración propia

Para la etapa de entrenamiento se utilizó el código "entrenamiento_y_envio.py" el cual se encargó con las librerías OpenCv y NumPy del procesamiento de las imágenes obtenidas, las cuáles entrarán en un proceso de detección y parametrización que genera como resultado un archivo con extensión. Yml, el cual contiene la data procesada de las fotos obtenidas dando como resultado el reconocimiento paramétrico de un rostro, tal como se muestra en la figura 9.

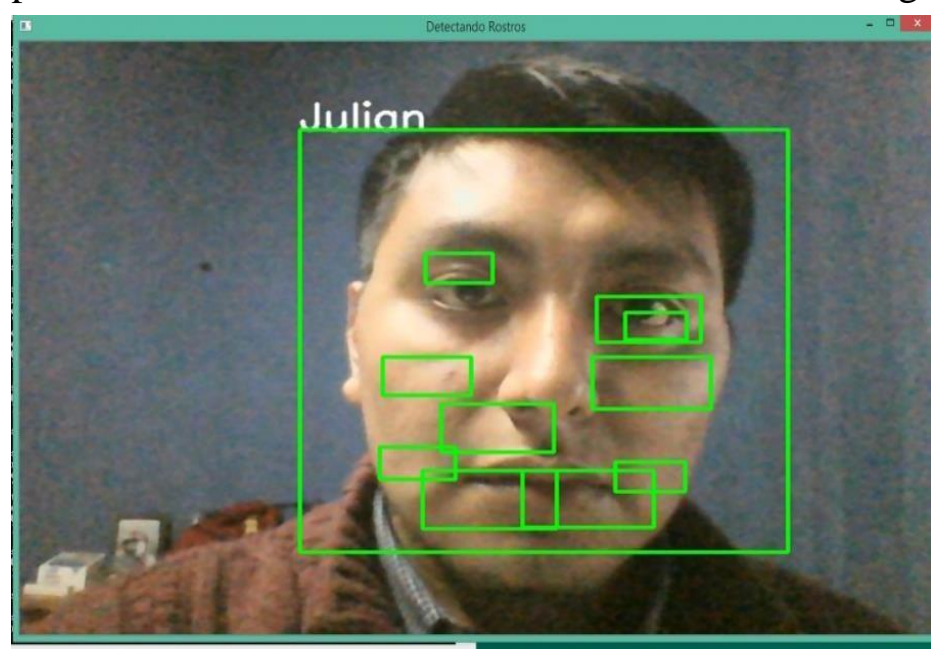

Figura 9. Pruebas de reconocimiento de visión artificial Fuente: Elaboración propia

Para realizar la transferencia de archivos entre el microprocesador y el servidor se utilizó el protocolo SFTP (SSH File Transfer Protocol) el cual describe la transferencia de archivos común vía FTP pero BIOTECH \& ENGINEERING Untels. Ene - jun.1(1), 2021; ISSN: 2788 - 4295; 63 - 81 
utilizando los parámetros de $\mathrm{SSH}$; ante ello se realizaron pruebas preliminares para determinar la viabilidad de la transferencia de archivos vía Sockets TCP, tal como se muestra en la Figura 10.

Figura 10. Pruebas de emisión de archivos usando Socket TCP Fuente: Elaboración propia

La transferencia del archivo enviado a modo de prueba es casi inmediata. El archivo enviado a través de sockets es cambiado de nombre (prueba_exitosa) al recibirse en la carpeta como se denota en la figura 11 .

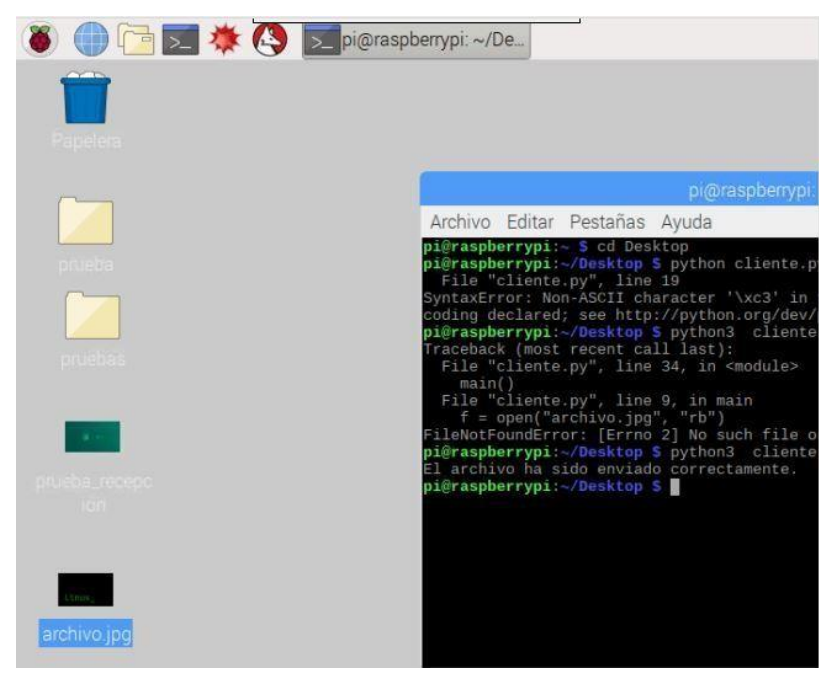

\begin{tabular}{|c|c|c|c|}
\hline Nombre & Fecha de modifica... & Tipo & Tamaño \\
\hline iㅣㄹ Avance de informe & $19 / 11 / 201913: 59$ & Documento de Mi... & $8.296 \mathrm{~KB}$ \\
\hline 5 client & $15 / 09 / 201922: 22$ & Archivo PY & $1 \mathrm{~KB}$ \\
\hline D. prueba_exitosa & $20 / 11 / 201910: 01$ & Imagen PNG & $72 \mathrm{~KB}$ \\
\hline 5 server & $15 / 09 / 2019: 22: 22$ & Archivo PY & $2 \mathrm{~KB}$ \\
\hline S servidor & $20 / 11 / 20199: 53$ & Archivo PY & $1 \mathrm{~KB}$ \\
\hline
\end{tabular}

Figura 11. Pruebas de recepción de archivos

Fuente: Elaboración propia

Entre las bondades de SFTP está la encriptación y en algunos casos poca compatibilidad con algunos equipos, si bien es cierto puede parecer desventaja para el desarrollo del proyecto resulta provechoso dado que de esa manera es factible mermar en gran parte posibles ataques que pueda sufrir el sistema en conjunto. La recepción del archivo se realiza en un entorno Linux probando así la versatilidad del protocolo y la compatibilidad de los entornos de trabajo, la prueba de ello se denota el archivo de nombre "prueba_recepcion", tal como se muestra en la figura 12. 


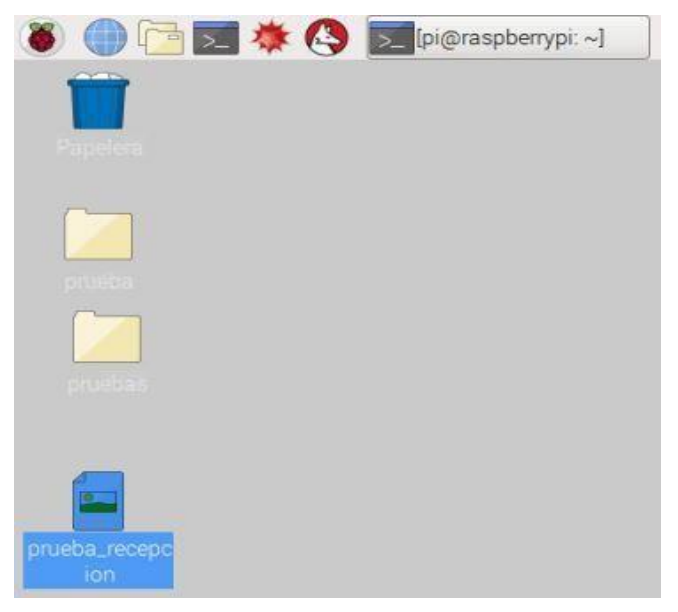

Figura 12. Recepción de imagen de prueba en plataforma Linux

Fuente: Elaboración propia

El análisis de la transferencia, con la finalidad de revisar la encriptación de la transferencia, se realizó el software libre Wireshark, que cuenta con herramientas para analizar el tráfico de una red, ante ello se analizó filtrando el puerto 22 de TCP el cual es utilizado para SSH y se obtuvo el resultado de la encriptación de la transferencia de data de manera exitosa, lo que se muestra en la figura 13.

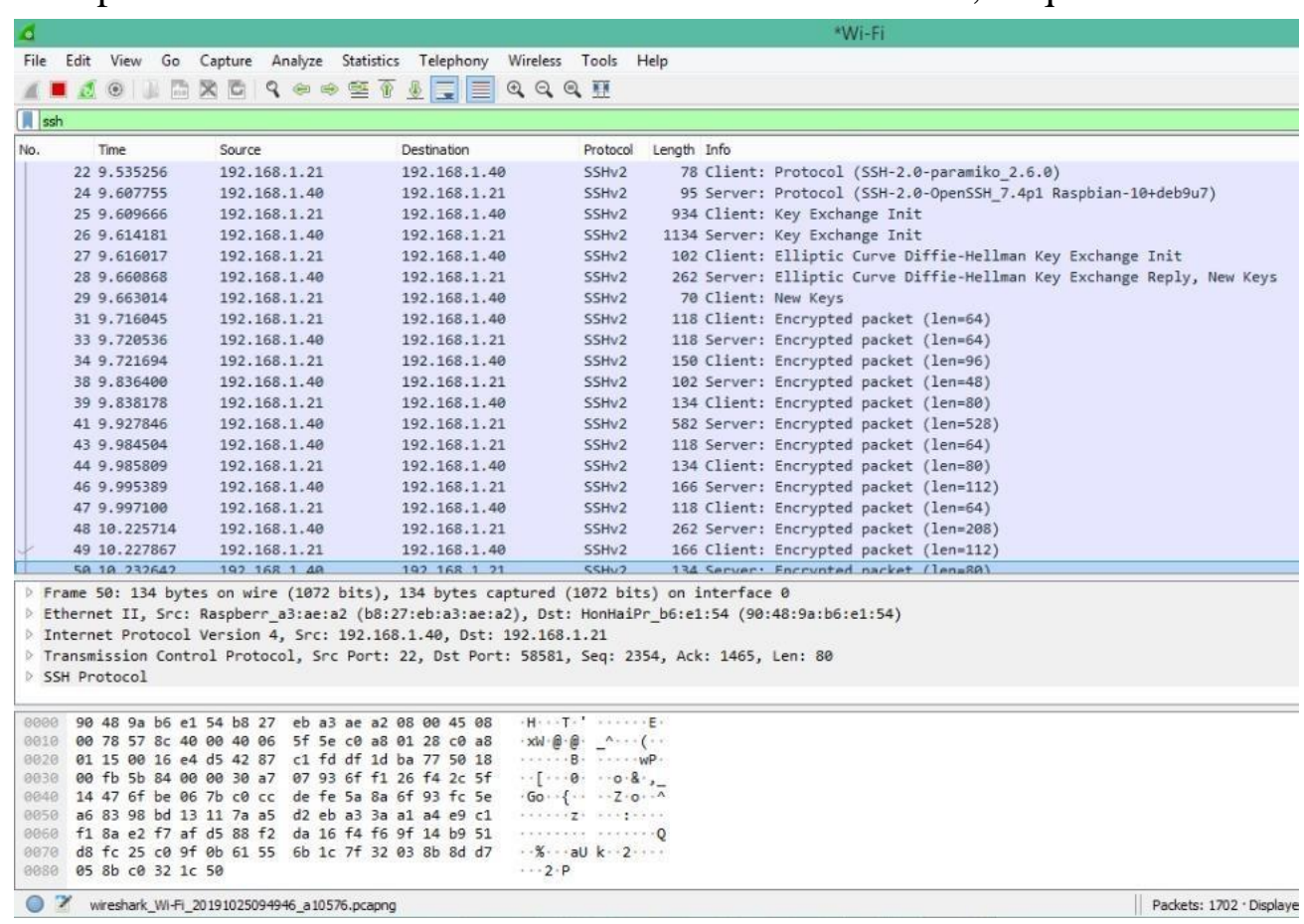

Figura 13. Análisis del tráfico de datos utilizando SFTP Fuente: Elaboración propia

\section{RESULTADOS Y CONTRIBUCIONES}

BIOTECH \& ENGINEERING Untels. Ene - jun.1(1), 2021; ISSN: 2788-4295; $63-81$ 


\section{Pruebas y resultados de la investigación}

Se detalla las pruebas realizadas y resultados obtenidos a través de la simulación del diseño del sistema mecánico y electrónico, así como del sistema de Red y Cloud local.

\section{Simulación del diseño del sistema mecánico - electrónico}

La simulación del movimiento del cuello del robot humanoide entrega resultados favorables para los movimientos de giro hacia la derecha e izquierda, tal como se muestra en la figura 14.

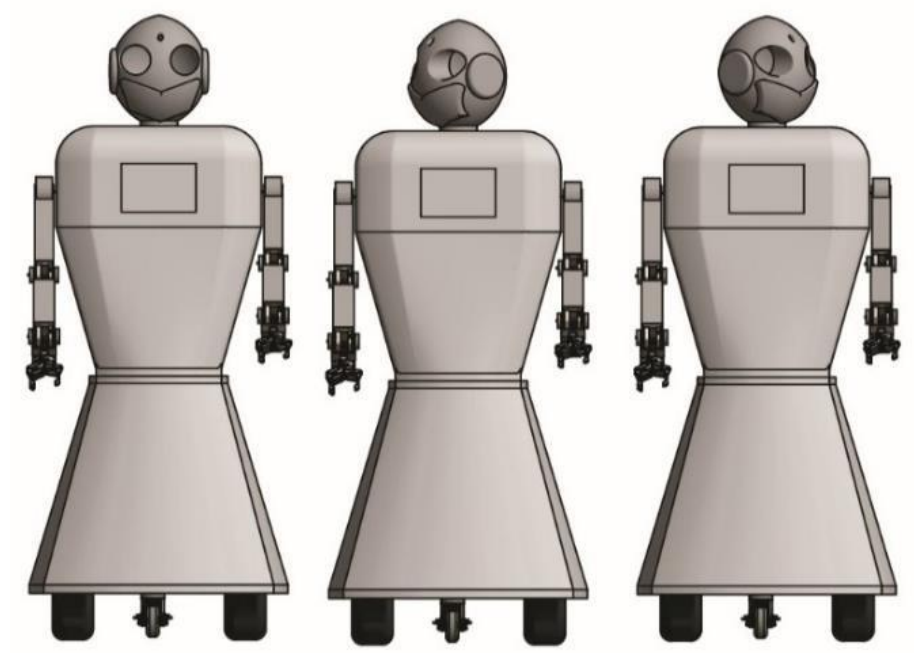

Figura 14. Simulación del movimiento del cuello del robot humanoide Fuente: Elaboración propia

A través de la simulación del diseño del sistema mecánico - electrónico diseñado para el movimiento de la garra del robot humanoide se obtiene resultados favorables para movimientos de apertura y cierre de la garra, tal como se muestra en la figura 15. 

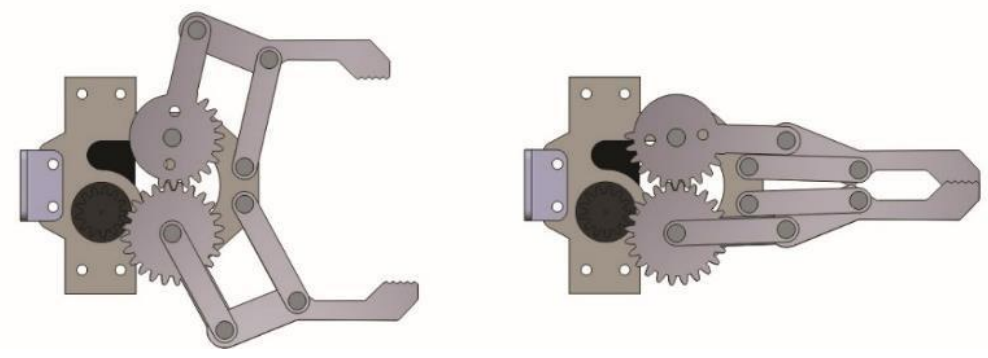

Figura 15. Simulación del movimiento de la garra del robot humanoide Fuente: Elaboración propia A través de la simulación del diseño del sistema mecánico - electrónico diseñado para el movimiento de los brazos del robot humanoide se obtienen resultados favorables para movimientos del brazo hacia arriba, tal como se muestra en la figura 16.

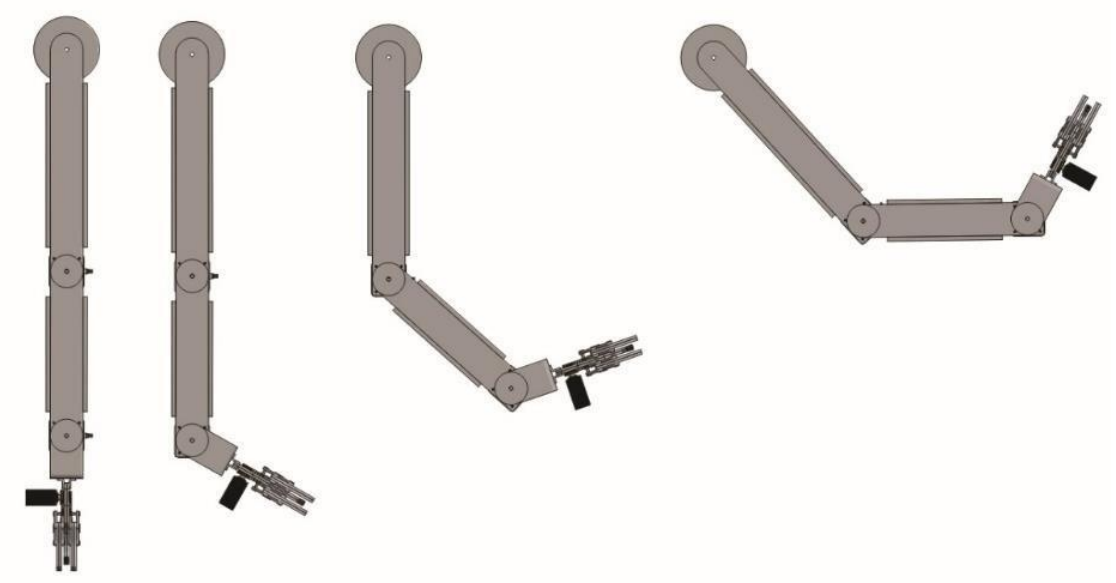

Figura 16. Simulación del sistema mecánico - electrónico de movimiento de los brazos del robot humanoide

Fuente: Elaboración propia

A través de la simulación del diseño del sistema mecánico - electrónico diseñado para el movimiento de traslación del robot humanoide se obtienen resultados favorables para movimientos del robot humanoide hacia adelante y hacia atrás, tal como se muestra en la figura 17. 

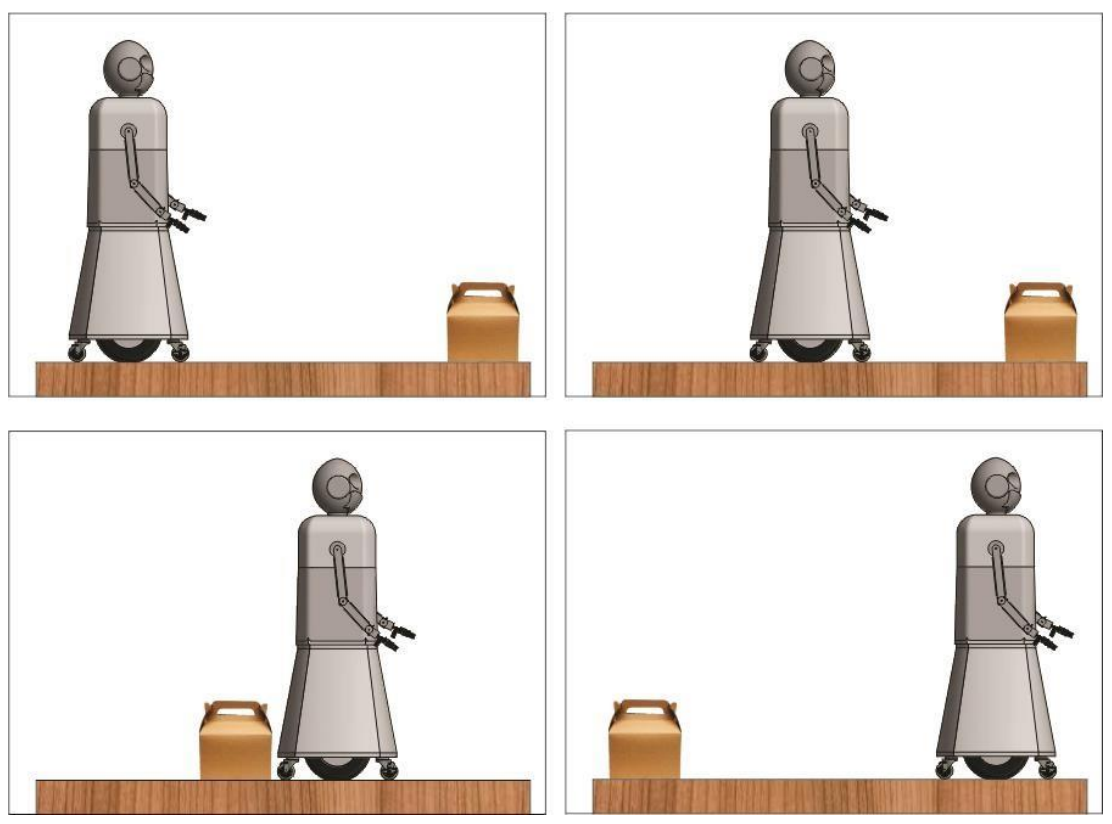

Figura 17. Simulación del sistema mecánico - electrónico de movimiento del robot humanoide Fuente: Elaboración propi 


\section{Simulación del diseño del Sistema de Red y Cloud local. Pruebas de reconocimiento facial}

Para las pruebas se realizó un entorno con luminosidad y en otros con una baja exposición de luz para realizar los descartes debidos y corroborar que el algoritmo trabaja de manera correcta y se mantiene tal cual lo establecido. El algoritmo se probó con la imagen de uno de los colaboradores, Julian Ruiz, se obtuvo el resultado deseado ya que el algoritmo entrega como resultado el nombre del colaborador, demostrado de esta manera, que reconoce los parámetros de los rasgos faciales, tal como se muestra en la figura 18.

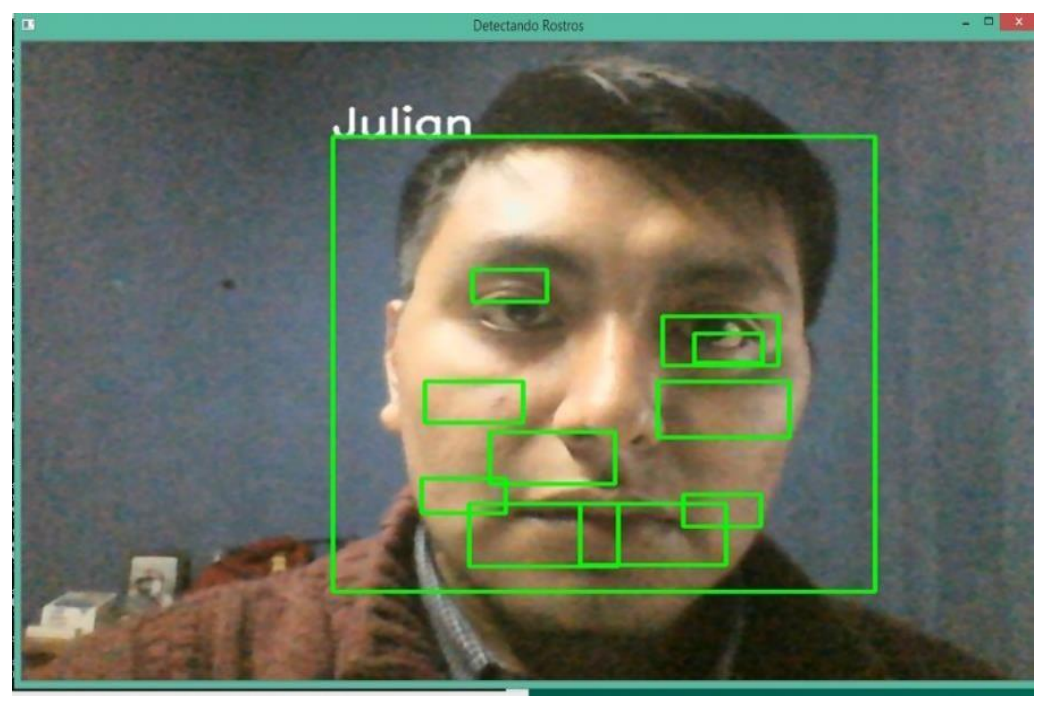

Figura 18. Pruebas de reconocimiento de rostro de colaborador

Fuente: Elaboración propia

\section{Contribuciones de la investigación}

La presente investigación contribuyó con la comunidad académica al diseñar y simular un Robot Humanoide de Servicio que permita el logro de las competencias de los estudiantes usuarios de los Laboratorios de Control - Automatización y Telecomunicaciones de la Universidad Nacional Tecnológica de Lima Sur.

Así mismo, contribuye con la comunidad científica de la Universidad Nacional Tecnológica de Lima Sur al brindar una plataforma para la aplicación de tecnologías IoT, Robótica e inteligencia artificial para futuras investigaciones. 


\section{CONCLUSIONES}

Se diseñó el sistema mecánico - electrónico de un robot humanoide de manera exitosa a través de la determinación de los materiales de sus partes, el dimensionado de los mismos, la selección de su procesador, controlador y sensores y periféricos de entrada y salida garantizando así la funcionabilidad del robot humanoide.

Se diseñó el Sistema de Red y Cloud local del robot humanoide de manera exitosa a través de la selección y configuración de un Servidor web, así como, la elaboración y entrenamiento del software de reconocimiento de rostro.

Se simuló el sistema mecánico, electrónico y de Red - Cloud local del robot humanoide de manera exitosa a través del uso de softwares que permitan obtener un entorno virtual de movimientos de cuello, brazos y traslación del robot humanoide, así como la transferencia y encriptación de Archivos, así como del algoritmo de reconocimiento de rostro. 


\section{REFERENCIAS}

Aracil R. (2002). Teleoperación. III Jornadas de Trabajo Enseñanza vía Internet/Web de la Ingeniería de Sistemas y Automática.

Ángeles, J. (2007). Fundamentals of Robotic Mechanical Systems. Theory, Methods and

Algorithms". Springer-Verlag.

Barrientos, A. (2007). Fundamentos de robótica. McGraw-Hill/ Interamericana de España.

Blanco. D, S. Ansari, C. Castejon, B. López, L. Moreno (2005), "Manfred: Robot Antropomórfico de servicio fiable y seguro para operar en entornos humanos", Revista Iberoamericana de Ingeniería Mecánica, Vol. 9, N³, pp. 33-48, España.

Cabrera, R., Chavez, P. (2013). Diseño e implementación de un robot humanoide asistencial controlado por computador para aplicaciones en pacientes parapléjicos. Universidad Ricardo Palma. http://repositorio.urp.edu.pe/handle/urp/403.

Corke P. (2011). Robotics, Vision and Control: Fundamental Algorithms in MATLAB.

Springer.

Craig, J. (2005). Introduction to Robotics, Mechanics and Control. Pearson Prentice Hall. De Hoz D. \& Saldana J. Decoupling Security from Applications for a Safer IoT.

Fong T. \& Nourbakhsh I \& Dautenhahn K. (2002). A Survey of Socially Interactive Robots: Concepts, Design, and Applications. The Robotics Institute Carnegie Mellon University, Report CMU-RI-TR02-29.

Gallardo C. \&. Sánchez Q. Diseño y construcción de un sistema de autentificación con reconocimiento facial mediante procesamiento de imágenes con la utilización de software libre y tecnología Raspberry PI.

Hernández. C, Montoya. C (2007), "Diseño de un sistema emulando el movimiento articulado de una mano, brazo y antebrazo", Tesis de Maestría en Ingeniería Mecatrónica, CENIDET, México. https://dialnet.unirioja.es/descarga/articulo/5732977.pdfJardón. A (2006), "Metodología de diseño de robots asistenciales. Aplicación al robot portátil ASIBOT", Tesis Doctoral dirigido por A. Giménez, C. Balaguer. Universidad Carlos III de Madrid, España.

Khamis R. (2003). Interacción remota con robots móviles basada en Internet.

http://citeseerx.ist.psu.edu/viewdoc/download?doi=10.1.1.100.6237\&rep=rep1\&type

BIOTECH \& ENGINEERING Untels. Ene - jun.1(1), 2021; ISSN: 2788 - 4295; 63 - 81 
$=\mathrm{pdf}$

Loza. E (2008), "Brazo Robótico controlado por una computadora en un ambiente de realidad virtual", Tesis Maestría en Ciencias en Tecnología de Computo, IPN, México. https://tesis.ipn.mx/jspui/bitstream/123456789/4047/1/BRAZOROBOTICO.pdf

Mohannad A. \& Abuzneid-Ausif M. Enhanced Human Face Recognition Using LBPH Descriptor, Multi-KNN, and Back-Propagation Neural Network. https://ieeexplore.ieee.org/document/8334532

Mohammad N. (2010)._Modeling and Simulation In SIMULINK for Engineers and

Scientists.MathWorks

Reyes F. (2011). Robótica: Control de Robots Manipuladores. Alfaomega.

Sabater Navarro, J. (2012). Guía Docente para el Diseño de Robots de Servicio. Instituto

Tecnológico de Construcción.

Sheridan T. (1997). Eight Ultimate Challenges of Human-Robot Communication. In

Proceedings RO-MAN, IEEE, Piscataway.

Siciliano, B., \& Khatib, O. (2017). Handbook of Robotics. Napoles: Springer. 